\title{
Towards an Intelligent Personalized Persuasive Conversational System for Human Interaction on Divining the Future Event and Assisting by Using Artificial Intelligence
}

\author{
Sweta Sharma ${ }^{\# 1}$, Saumya Pathak ${ }^{\# 2}$, Saumya Srivastava ${ }^{\# 3}$

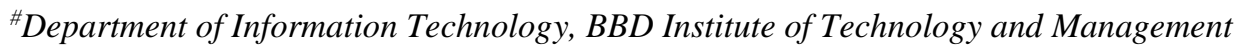 \\ Lucknow, India \\ ${ }^{1}$ sh. sweta.xtabbdnitm.ac.in \\ ${ }^{2}$ harshpathak100@bbdnitm.ac.in \\ ${ }^{3}$ saumyasrivastava7272@bbdnitm.ac.in
}

\begin{abstract}
In the next wave of insurgence, humans may endeavour self-reflection which can lead to an effortless talk and to find out if an event will fructify. Training the system on how to make accurate prognostication with the help of machine learning and statistical models can lead to an intelligent personalized conversational system. The Chatbot industry is ever-growing and after the COVID-19 pandemic and rigorous lockdowns all around the world, people have realized the importance of human interaction in their lives. We are developing this model to create a more intimate relationship between the system and humans. For this purpose, many open-source platforms are available. Artificial Intelligence Markup Language (AIML) is derived from Extensible Markup Language (XML) which is used to build up a conversational agent artificially. The success of this project will help the model in observing and understanding human emotions which will ultimately help it to form a more personalized relationship to delineate the future course of events.
\end{abstract}

Index Terms - Prognostication, Machine Learning, Statistical Model, Chatbot, Artificial Intelligence, AIML, Future

\section{INTRODUCTION}

Across cultures and history, we discover individuals wanted to grasp their future. People are continually attempting to seek out their own life to know how badly things can go and to decide how much work they need to put into it to change unusual things into satisfying outcomes in the future.

Moreover, the personal information of humans would affect moral suasion. Carl Rogers believed that all people can bring about positive change in their lives. Rogers' approach to psychotherapy is considered humanistic because it focuses on people' positive potential. Another important aspect of future prognostication is good judgement. It combines both knowledge and strategies related to the outcome of interest to achieve better results ${ }^{[1]}$. Large-scale conversational AI agents such as Alexa, Siri, and Google Assistant are becoming more and more prevalent, opening up in new domains and taking up new tasks to serve users across the globe.

Our model will achieve this by understanding human behaviour and psychology so that it can converse fluently with humans without the stiffness of a bot. One key consideration in designing such systems is how they can be improved over time at that scale. Users interacting with these agent's experience frictions due to various reasons.

\section{RELATED WORK}

A prediction is usually based on experience or knowledge. In the past predictions have often been made by using paranormal or supernatural means such as prophecy or by observing omens. Methods including astrology, numerology, fortune-telling, water divining, interpretation of dreams, and lots of other modes of divination, have been used to attempt to prognosticate.

But in the Foundation Trilogy series by Isaac Asimov, a mathematician finds out that historical events can be theoretically modelled using equations, and then spends years trying to put the theory into practice. The modern science of psychohistory founded upon his success can simulate history and extrapolate the present into the future.

Thus, studying human behaviour to predict how people will behave and consequently what is going to happen in their future makes complete sense. Early humans had to trust their instincts but what they didn't have was complete data to infer their future.

In recent years, computational power and methods of collecting data have advanced to an idea of creating a new field: Big Data. For example, Amazon uses predictive analytics which encompasses a variety of statistical techniques to guess which books we may like based on our previous browsing or purchase records. Furthermore, automated online advertisement campaigns tell us which vehicles we may be interested in based on vehicles sought out the day before ${ }^{[2]}$

Marketers use birth records to decide when to inundate people with ads for baby merchandises. They even guess when they would need their products based on the growth of their child. Various prediction tools rely on machine learning, among which include mathematical algorithms that are based on the biological principles of brain reception and use huge amounts of data to learn patterns. On the other hand, Sciences 
like futurology and meteorology also deal with the future. Now, what distinguishes astrology from these other futurepredicting subjects is that astrology considers the movements of the nine planets across the zodiac in making prognostications. Futurologist looked beyond the vision to forecast outcomes by studying the universe. Prediction of the past is the vital first step of science to determine the future. The next step is whether your new theory continues to explain the world as new data comes in. A key challenge in prognosticating is the lack of data and research on human brain activities ${ }^{[2]}{ }^{[3]}$.

With more numerous data, accuracy continually increases and uncertainty goes down. We are not talking about a vague, ambiguous prediction, but reasoned cautious and thoughtful foresight.

\section{PROCEDURES}

Before the conversation began, we ask the user to complete the form to assess their name and birth details. Next, we open up the channel to the user to interact with the system. It performs a wide range of assistive features like PDF to audio conversion, web article reader (learn by listening), searching queries and videos, launching applications, setting a reminder, word meaning and definitions.

Our system does this by using some useful APIs and datasets in a friendly and conversational manner making the user more at ease. We choose to predict the most salient strategy for each human conversations to understand their behaviour.

\section{A. AIML Version 1.0}

The primary design goal of the original AIML (Artificial Intelligence Markup Language) language was simplicity. It is easy to learn and implement. It also facilitates the userfriendliness of the system.

AIML illustrates a correlation between three entities: the client, the botmaster (a human chatbot author), and the bot ${ }^{[4]}$.

Our system response could be from categories within its knowledge base. Listing 1. Shows code for accessing external resources.

\section{Listing 1.}

$<$ category>

$<$ pattern $>$ WHAT IS MEANT BY $*<$ pattern $>$

$<$ template $><$ srai $>$ WHAT IS THE MEANING OF

$\langle$ star $/\rangle\langle$ srai $\rangle\langle/$ template $>$

$\langle/$ category $>$

$<$ category >

$<$ pattern $>$ WHAT MEANS BY *</pattern $>$

$<$ template $><$ srai $>$ WHAT IS THE MEANING OF

$<$ star $/></$ srai $></$ template $>$

$</$ category $>$

$<$ category $>$

$<$ pattern $>$ WHAT IS $*<$ pattern $>$

$<$ template $><$ srai $>$ WHAT IS THE MEANING OF

$<$ star $/><$ srai $></$ template $>$

$</$ category $>$

$<$ category >

\section{<pattern>WHAT DOES IT MEAN BY $*<$ pattern> $<$ template $><$ srai $>$ WHAT IS THE MEANING OF $\langle$ star $/\rangle\langle$ srai $\rangle</$ template $>$ \\ $</$ category $>$}

The category element defines a question-response pair; the question is defined within the pattern tag pair (<pattern $></$ pattern $>$ ) and the response in the template tag pair $(<$ template $></$ template $>$ ). The $<$ star $/>$ tag is used to retrieve variables (denoted by $*$ ) provided by the users to the chatbot ${ }^{[4]}$.

\section{B. AIML Version 2.0}

The chatbot market has grown and emerged since AIML 2.0 was released four years ago, and this spec update injects some new key features designed to address the changing landscape. Pattern matching was one of the main improvement in this version.

AIML 2.0 is an effort to address the shortcomings, while balancing the primary goal of keeping the language as simple as possible ${ }^{[4]}$.

Some of the new features added in AIML 2.0 are listed below.

- Highest priority matching

- AIML Sets

- AIML Maps

- Loops

- Local variables

- Sraix

- Denormalization

- Out of Band Tags

\section{CONCLUSIONS}

One of the foremost powerful influences on fear is uncertainty. The less we know, the more threatened we feel. This system ensures to provides the user with a feeling of certainty over their future and reduces their anxiety and the fear of the unknown. It does this in a friendly and conversational manner making the user more at ease.

We focus on developing a model which will be able to generate responses automatically to the questions asked using several various machine learning techniques. Humans will endeavour self-reflection with the system which can lead to an effortless talk and to find out if an event will fructify. The proposed model could be used for basic assistive features and also providing a more probabilistic approach to the mystery of the future.

\section{ACKNOWLEDGMENT}

We would like to express our sincere appreciation to Professor Asit Kumar Gahalaut for his patient guidance, enthusiastic encouragement and useful critiques of this research work. His invaluable suggestions and practical advice helped us tremendously during our research and planning of this project.

We would like to offer our special thanks to our institute for offering us the resources. 
We would also like to acknowledge our parents, who have given us moral and unwavering support every time.

Thanks for all your encouragement!

\section{REFERENCES}

[1] Cooper, M., Watson, J. C., \& Hoeldampf, D. "Rogerian and experiential therapies work: A review of the research on psychotherapy, counselling and related practices," Ross-on-Wye, UK: PCCS Books 2010, chapter 5-7.

[2] Mohammad Hossein Jarrahi, "Artificial Intelligence and the Future of Work: Human-AI Symbiosis in Organizational Decision Making," 2018, pp. 5-10.

[3] Janna Anderson, Lee Rainie, "Improvements ahead: How humans and AI might evolve together in the next decade," Dec. 2018.

[4] (2018) AIML Docs - "AIML Foundation" [Online]. Available: http://www.aiml.foundation/doc.html/ 